\title{
Dictionary Indexing of Electron Channeling Patterns
}

\author{
Saransh Singh, and Marc De Graef* \\ Department of Materials Science and Engineering, Carnegie Mellon University, 5000 Forbes Avenue, Pittsburgh, \\ PA 15213, USA
}

\begin{abstract}
The dictionary-based approach to the indexing of diffraction patterns is applied to electron channeling patterns (ECPs). The main ingredients of the dictionary method are introduced, including the generalized forward projector (GFP), the relevant detector model, and a scheme to uniformly sample orientation space using the "cubochoric" representation. The GFP is used to compute an ECP "master" pattern. Derivative free optimization algorithms, including the Nelder-Mead simplex and the bound optimization by quadratic approximation are used to determine the correct detector parameters and to refine the orientation obtained from the dictionary approach. The indexing method is applied to poly-silicon and shows excellent agreement with the calibrated values. Finally, it is shown that the method results in a mean disorientation error of $1.0^{\circ}$ with $0.5^{\circ} \mathrm{SD}$ for a range of detector parameters.
\end{abstract}

Key words: electron channeling pattern, ECP, dynamical electron scattering, dictionary matching

\section{INTRODUCTION}

Electron channeling was first observed in a scanning electron microscope (SEM) by Coates in (1967). The channeling contrast arises from the variation of the backscattered electron yield as a function of the incident beam direction which manifests itself in the form of a "Kikuchi-like" band pattern. Practical applications of electron channeling patterns (ECPs), including crystallographic orientation mapping, measuring lattice bending, etc. have been proposed (Joy et al., 1982). Electron channeling has also been used extensively for the characterization of extended defects in a variety of material systems (Ahmed et al., 1997; Trager-Cowan et al., 2007; Picard \& Twigg, 2008; Mansour et al., 2015; Deitz et al., 2016). Historically, channeling patterns were acquired by simply running the instrument at the lowest possible magnification and a high-beam current. In this mode, a Kikuchi-like band pattern is observed superimposed on the normal topographic image. The main drawback of this method is the poor spatial resolution. Hence, only single crystal or large grained microstructures were amenable to this technique. Recent advances in electron optics have led to the development of the selected area channeling pattern (SACP) mode, for which the pivot point of the electron beam lies on the sample surface (Van Essen \& Schulson, 1969; Van Essen et al., 1970; Joy \& Booker, 1971). This has improved the spatial resolution by many orders of magnitude and is now available commercially.

ECP-based defect characterization is referred to as electron channeling contrast imaging (ECCI) and offers some advantages compared with defect characterization in a transmission electron microscope (TEM), including easier sample preparation and the ability to study bulk samples. Quantitative study of defects is also possible in ECCI mode

Received October 31, 2016; accepted December 20, 2016

*Corresponding author. degraef@cmu.edu using some of the same principles as used for TEM analysis. Examples include application of the $\mathbf{g} \cdot \mathbf{b}$ and $\mathbf{g} \cdot(\mathbf{b} \times \mathbf{u})$ invisibility criteria for dislocations and the $\mathbf{g} \cdot \mathbf{R}$ criterion for stacking faults (Czernuszka et al., 1990; Crimp et al., 2001). However, such analyses require knowledge of the crystal orientation to determine the reciprocal lattice vectors $\mathbf{g}$ corresponding to the bands in the ECP. Although there have been efforts to combine the existing electron backscatter diffraction (EBSD) technique with ECCI to accomplish this (Gutierrez-Urrutia et al., 2013; Zaefferer \& Elhami, 2014), this approach requires extra hardware and is not available as a commercial product. Henceforth in this paper, assigning an orientation to a diffraction pattern using one of the many available representations (e.g., Euler angles, Rodrigues vector, quaternions, etc.) will be referred to as "indexing" the diffraction pattern.

ECP and EBSD patterns are related to each other through the reciprocity theorem. However, the indexing of ECPs using the usual Hough transform-based method is difficult due to the limited angular range of the pattern. Although the Hough transform can be used to index ECPs for certain special cases (Schmidt \& Olesen, 1989), for example, close to a low-index zone axis, there is no general framework which can be used to index an arbitrary ECP. The dictionary-based method has been shown to successfully index EBSD patterns using a library of precomputed patterns which have been uniformly sampled from the Rodrigues fundamental zone (FZ) of the crystal under consideration (Chen et al., 2015).

In this paper, we propose a similar dictionary-based algorithm to index ECPs, which will serve as a general framework for the indexing of channeling patterns, irrespective of the angular range of the illumination. As the dictionary approach uses all the available pixels in the image instead of only the linear features, this method is more robust to noise in the experimental patterns than the Hough transform method 
(Wright et al., 2015). We begin with a discussion of the general framework of this approach; the various ingredients, which include a forward model for dynamical diffraction, are introduced, as well as the incorporation of the common types of noise and lens aberrations. Then we describe both detector parameter estimation and orientation refinement using a derivative free optimization (DFO) algorithm. A new scheme to uniformly sample orientation space using the "cubochoric" projection is briefly discussed. Then, we discuss results of our approach for the indexing of ECPs of a model system, namely polycrystalline silicon (poly-Si). We also describe the sensitivity of the method to the various input parameters. Finally, we conclude this paper with a brief summary.

\section{Dictionary-Based Indexing Approach}

Dictionary-based indexing refers to a wide class of algorithms that employ a library of precomputed patterns to index experimental diffraction patterns. Instead of extracting features, such as bands, spots etc., from the experimental patterns, the dictionary approach uses all the available pixels in the pattern to find a best match with respect to the dictionary patterns. There are three necessary ingredients to generate this library: (1) a generalized forward projector (GFP), which is a physics-based forward model of the scattering process; (2) an accurate detector and noise model; and (3) a method to uniformly sample orientation space, $\mathrm{SO}(3)$. Although the GFP and the detector and noise model will change for different diffraction modalities, the method to uniformly sample orientation space is common to all modalities; this has been discussed in detail in (Singh \& De Graef, 2016). The three ingredients combined with a pattern matching engine (Goshtasby, 2012) (cross-correlation, mutual information, etc.) form the basis of the dictionarybased approach to indexing diffraction patterns. In the remainder of this section, we will discuss in detail each aspect of the dictionary-based approach as it is adapted to the ECP modality. A schematic for dictionary-based indexing of ECPs is shown in Figure 1.

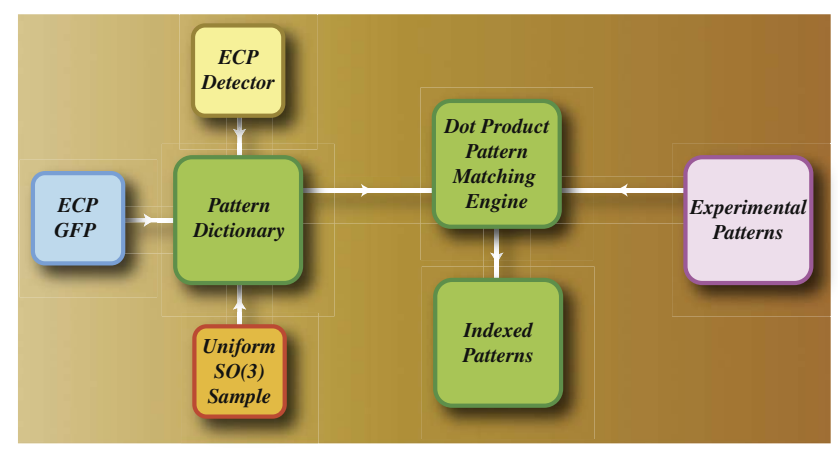

Figure 1. Schematic representation of the electron channeling patterns (ECP) dictionary indexing process. Arrows represent the flow of data/information. GFP, generalized forward projector.

\section{Forward MOdel fOR ECPs}

The GFP described in this section has three main components: dynamical scattering of electrons, Monte Carlo (MC) simulations to generate spatial and depth statistics, and a realistic detector, noise and distortion model. In this section, we will briefly consider each of these components to generate a computational framework for the simulation of ECPs. The dynamical scattering accounts for the elastic channeling of the electrons by the crystal potential, whereas the MC simulation accounts for the stochastic nature of inelastic scattering. The MC simulations also provide the backscattering depth statistics, which are needed to set the integration depth for the dynamical simulations. The two approaches are merged together to compute a "master channeling pattern" that represents the diffracted intensity distribution on a spherical surface surrounding the crystal. The pattern is stored as a square intensity grid, using a modified Lambert equal-area projection (Roşca, 2010). The master pattern can be sampled for a given sample and detector geometry, as well as a given crystal orientation, which can be specified as a triplet of Euler angles, a unit quaternion, etc. A similar computational approach has already been used for the simulation of EBSD patterns (Callahan \& De Graef, 2013). Such a GFP is also necessary to make the dictionary indexing method computationally tractable as a large number of dynamical diffraction patterns need to be calculated for reliable indexing. The master pattern for Silicon using the modified Lambert projection along with the stereographic projection are shown in Figures $2 \mathrm{a}$ and $2 \mathrm{~b}$, respectively. In the next subsection, we will briefly outline the dynamical scattering process and the Bloch wave method used to compute the BSE amplitude for different channeling directions, as well as the detector model. Finally, we conclude the section by discussing the relevant noise model and electron optical aberrations.

\section{Dynamical Diffraction}

A number of theoretical models have been proposed to explain channeling contrast as a function of incoming beam direction with respect to the crystal reference frame (Spencer \& Humphreys, 1980; Marthinsen \& Høier 1986; Rossouw et al., 1994; Dudarev et al., 1995; Winkelmann et al., 2003). For the present study, we focus on the theoretical model outlined in Picard et al. (2014), which distinguishes between BSE1 and BSE2 electrons; BSE1 electrons undergo a backscatter event as their very first scattering event and are responsible for the channeling contrast. These electrons have nearly the same energy as the incident electrons as they immediately backscatter. The BSE2s, on the other hand, backscatter after undergoing multiple elastic and inelastic scattering events. These electrons carry no channeling information and form the background intensity. It has been shown in (Picard et al., 2014) that for a reasonable detector geometry, the typical ratio of BSE1s to BSE2s is of the order 

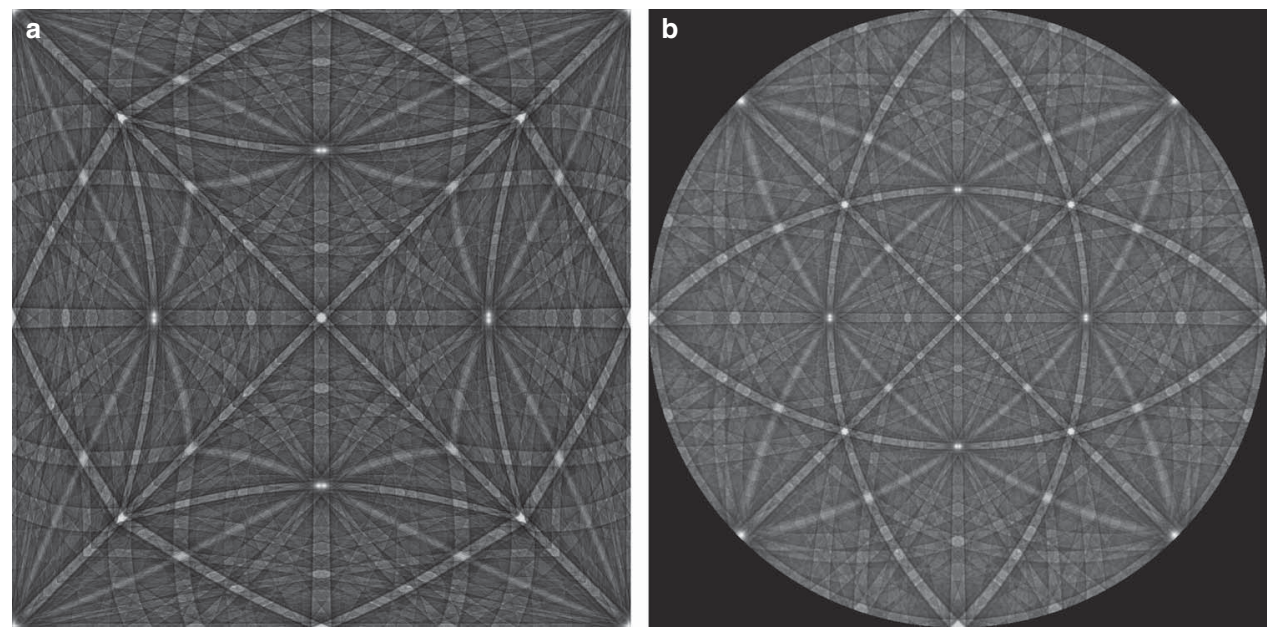

Figure 2. Master electron channeling pattern for $20 \mathrm{keV}$ beam acceleration voltage for Si using (a) modified Lambert projection and (b) stereographic projection.

of $10^{-3}-10^{-4}$. Therefore, to detect the weak BSE1 signal superimposed on top of the large BSE2 background signal, the brightness and contrast settings of the microscope have to be adjusted accordingly. Most of the BSE2 signal is usually removed by adjusting pattern brightness and contrast such that the dominant signal results from BSE1 electrons. Therefore, in the remainder of this paper, we will only focus on the BSE1 electrons.

The BSE1s can be generated from a range of depths inside the sample. Consequently, the overall signal will be a sum of the signals coming from different depths. Note that the incident electron channels on its way into the crystal, which is different from the EBSD technique, where the electron channels on its way out of the crystal. The range of depths from which the BSE1 signal arises can be estimated by means of MC simulations. There will also be normal and anomalous absorption of the BSE1 electrons as they channel through the crystal. This absorption is described phenomenologically by the imaginary part of the lattice potential and a depth dependent weight factor, whereas integrating the signal from various depths. For a crystal structure with $N_{a}$ atoms in the unit cell distributed over $n$ sites in the asymmetric unit, with a set $S_{n}$ of equivalent atom positions, we can write the overall BSE1 signal for a channeling direction described by the wave vector $\mathbf{k}_{0}$ as

$$
\mathcal{P}\left(\mathbf{k}_{0}\right)=\sum_{n} \sum_{i \in S_{n}} \frac{Z_{n}^{2} D_{n}}{z_{0}} \int_{0}^{z_{0}} \lambda(z)\left|\Psi_{\mathbf{k}_{0}}\left(\mathbf{r}_{i}\right)\right|^{2} d z
$$

where $Z_{n}$ and $D_{n}$ are the atomic number and Debye-Waller factor, respectively, of the atom at site $n$; $z_{0}$ is the depth of integration; $\lambda(z)$ is a depth dependent weight factor, and $\Psi_{\mathbf{k} 0}\left(\mathbf{r}_{i}\right)$ is the wave function of the electron which is channeling in the direction $\mathbf{k}_{0}$. The electron wave function is evaluated using either the Bloch wave or the scattering matrix approach (De Graef, 2003); in this paper, we restrict our discussion to the Bloch wave approach, in which the electron wave function is written as a superposition of Bloch waves traveling in different directions $\mathbf{k}^{(j)}$

$$
\Psi(\mathbf{r})=\sum_{j} \alpha^{(j)} \sum_{g} C_{\mathbf{g}}^{(j)} e^{2 \pi i\left(\mathbf{k}^{(j)}+\mathbf{g}\right) \cdot \mathbf{r}} .
$$

Here, $\alpha^{(j)}$ is the excitation amplitude of the $j$ th Bloch wave and $C_{\mathbf{g}}^{(j)}$ is the Bloch wave coefficient of the $\mathbf{g}$ beam for that wave. The above two equations can be combined to obtain the overall BSE1 signal for an electron incident in the $\mathbf{k}_{0}$ direction. It can be shown that the result can be expressed as the sum of elements in a Hadamard product (denoted by the symbol o) of two matrices, $S$ and $L$. The matrix $S$ can be thought of as a structure factor, whereas the matrix $L$ encodes the dynamical diffraction information. The individual elements of $S$ and $L$ are given by

$$
\begin{aligned}
S_{\text {gh }} & =\sum_{n} \sum_{\mathrm{i}_{n}} Z_{n}^{2} D_{n} \mathrm{e}^{2 \pi \mathrm{i}(h-g) \cdot \mathrm{r}_{i}} ; \\
L_{\text {gh }} & =\sum_{j} \sum_{k} C_{\mathrm{g}}^{(j)^{*}} \alpha^{(j)^{*}} \mathscr{I}_{j k} \alpha^{(k)} C_{h}^{(k)} .
\end{aligned}
$$

Here, $\mathscr{I}_{j k}$ is defined as

$$
\mathscr{I}_{j k}=\frac{1}{z_{0}} \int_{0}^{z_{0}} \lambda(z) \mathrm{e}^{-2 \pi\left(\alpha_{j k}+\mathrm{i} \beta_{j k}\right) z} d z,
$$

where $\alpha_{j k}=q^{j}+q^{k}$ and $\beta_{j k}=\gamma^{j}-\gamma^{k}$; the complex numbers $\lambda^{j} \equiv \gamma^{j}+i q^{j}$ are the eigenvalues of the dynamical Bloch matrix with absorption taken into account. Finally, all of this can be put together to obtain the depth-integrated probability of backscattering of the electron traveling in the direction $\mathbf{k}_{0}$, which is given by

$$
\mathcal{P}\left(\mathbf{k}_{0}\right)=\left\langle\mathbf{u}^{T}|S \circ L| \mathbf{u}\right\rangle .
$$

Here, $\boldsymbol{u}$ is a column vector with all its entries equal to unity and o represents the Hadamard (element-wise) product of two matrices. The above equation is nothing but the sum of elements of the Hadamard product of $S$ and $L$. 


\section{Detector Model}

A channeling pattern is recorded by measuring the backscattered signal as a function of the incident beam direction. MC simulations for BSE1 electrons reveal that the majority of BSE1s are scattered at a large angle with respect to the incident beam direction, typically in the range $50-70^{\circ}$. Figure $3 a$ shows the stereographic projection of the BSE1 exit direction distribution for $20 \mathrm{keV}$ electrons scattered from a Si sample. A polar plot of the BSE1 intensity as a function of angle with respect to the sample normal is shown in Figure 3b. The maximum intensity is at an exit angle of $58.1^{\circ}$. As it is desirable to capture a large portion of the backscattered electrons to enhance the already weak BSE1 cumulative signal, an annular integrating backscatter detector is used, as shown in the schematic of Figure 4a. The inner and outer radii of the detector and the sample to detector distance, $\xi$ are adjusted such that the detector captures a significant fraction of the total backscattered electron flux. For defect imaging, on the other hand, the sample is usually tilted to be close to a Bragg orientation. As a result, the background intensity will no longer have rotational symmetry around the incident beam direction. This asymmetry can be taken into account when the dynamical backscatter intensities are merged with the BSE1 results from stochastic MC simulations.

The MC simulations are performed for a range of incident beam angles, typically from $0-20^{\circ}$ in steps of $1^{\circ}$, resulting in the BSE1 backscatter yield as a function of exit direction and incidence angle, $\mathbf{Y}(\theta, \hat{\mathbf{n}})$. The inner and outer radius of the annular detector, together with the working distance, are used to calculate the solid angle range over which the MC simulations need to be integrated. The detector is discretized with a polar and azimuthal angular step size of $1^{\circ}$, producing a set of exit directions $\left\{\mathbf{e}_{i}\right\}$. For every incidence angle, $\theta_{j}$ from $0-20^{\circ}$, the effective weight factor is then calculated by summing the backscatter yield over the calculated solid angle range; mathematically, we have

$$
w\left(\theta ; R_{\text {in }}, R_{\text {out }}, \xi\right)=\sum_{\left\{\mathbf{e}_{i}\right\}} \mathbf{Y}(\theta, \hat{\mathbf{n}}) .
$$

For a realistic detector geometry with inner radius, $R_{\text {in }}=5.0 \mathrm{~mm}$, outer radius, $R_{\text {out }}=13.0 \mathrm{~mm}$ and a working distance of $\mathrm{WD}=5.0 \mathrm{~mm}$, the fraction of electrons captured by the detector is shown in Figure $4 \mathrm{~b}$. The weight factor for
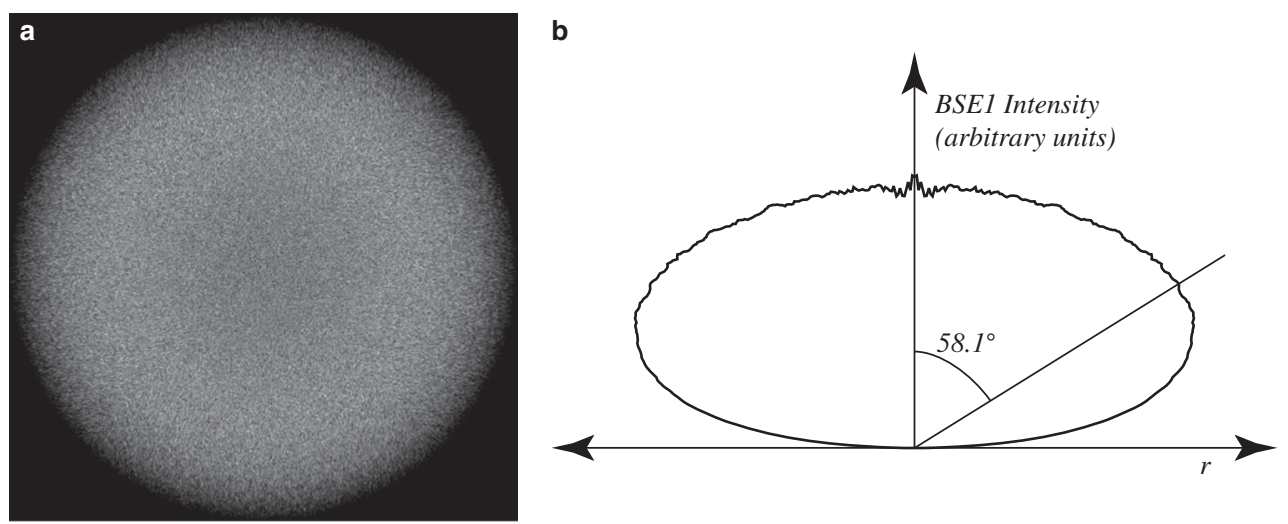

Figure 3. MC simulation for $20 \mathrm{keV}$ beam acceleration voltage for Si (a) stereographic projection of BSE1 electrons and (b) polar plot of backscatter yield as a function of angle from sample normal with the maximum at $58.1^{\circ}$.
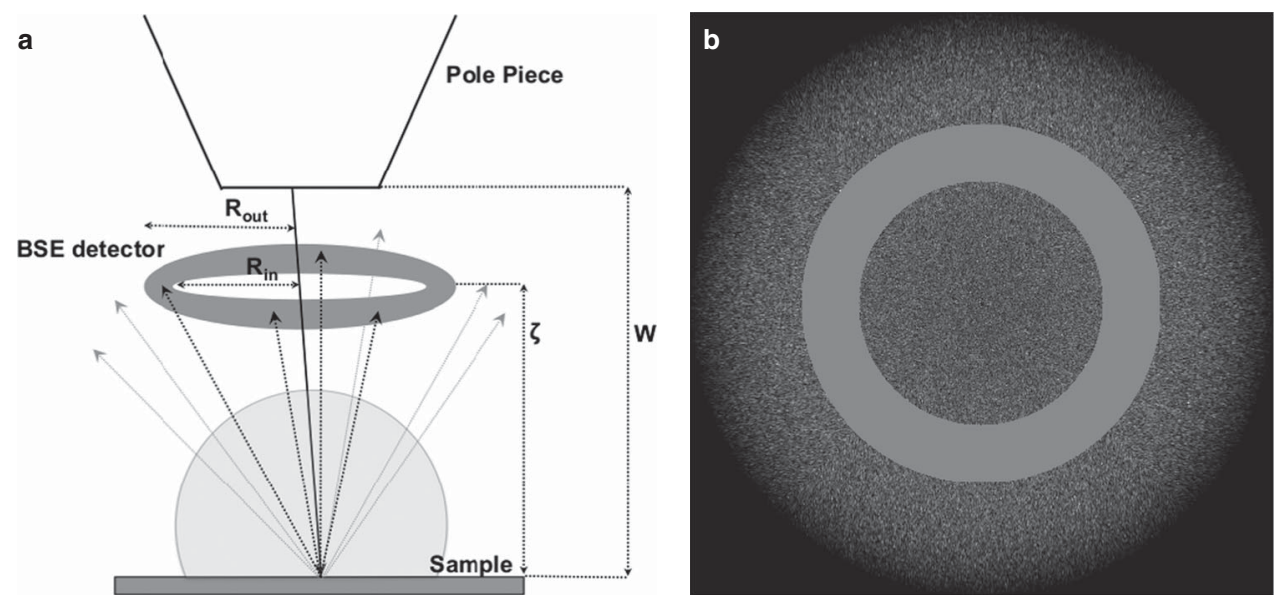

Figure 4. a: Schematic of the electron channeling patterns setup. Only a small fraction (black dashed) of the BSE1 electrons get captured by the annular detector and (b) stereographic projection of BSE1 exit direction for $20 \mathrm{keV}$ acceleration voltage on $\mathrm{Si}$ sample superimposed with the stereographic projection of all exit directions for which the electron is captured by the detector (detector geometry in text). 
an arbitrary incidence angle is calculated by linear interpolation using weight factors of the known angles. For a grain with orientation described by a unit quaternion, $\tilde{q}$, each pixel $(i, j)$ in the channeling pattern corresponds to an incident wave vector $\mathbf{k}_{i j}$ in the microscope reference frame; the intensity in the corresponding direction is interpolated from the MC and master patterns and is given by the expression

$$
I_{\mathrm{BSE}}=w\left(\theta ; R_{\mathrm{in}}, R_{\mathrm{out}}, \xi\right) \mathcal{M}\left(\mathbf{k}_{i j} ; \tilde{q}\right),
$$

where $\theta$ is the angle between the wave vector $\mathbf{k}_{i j}$ and the sample normal and $\mathcal{M}\left(\mathbf{k}_{i j}\right)$ is extracted from the master pattern using bilinear interpolation.

\section{Lens Aberrations and Noise}

Aberrations in the channeling patterns arise from the fact that the incident electron beam is scanned at a relatively large angle $\left(10-15^{\circ}\right)$ with respect to the optical axis. The primary (Seidel) aberrations of the objective lens (spherical aberration, coma, astigmatism, field curvature, and distortion) combine with the aberrations of the deflection system to introduce a significant amount of distortion in the highangle portion of the ECPs. We model the distortion in the following way: if $P_{i}$ represents a point in the image plane corresponding to a point $P_{0}$ in the object plane, then we assume that the location of $P_{i}$ is given by $u_{i}+\Delta u_{i}$, where $\Delta u_{i}=(D+i d)\left|u_{\mathrm{o}}\right|^{2} u_{\mathrm{o}} ; u_{0}$ is a complex number representing the location of point $P_{0}$, and $D$ and $d$ are the real and imaginary parts of the distortion coefficient. Depending on the sign of $D$, the distortion is either referred to as a barrel distortion $(D>0)$ or a pin-cushion distortion $(D<0)$. Note that the displacement field only depends on the position in the object plane, which makes the computation of the distortion field relatively straightforward. A full determination of the aberration field requires consideration of higher order terms. More details about this and various other SEM lens aberrations can be found in (Szilagyi, 1988; Hawkes \& Kasper, 1989b, 1989a).

Figure 5a shows an experimental channeling pattern for a semiconductor grade poly-Si sample with a large grain size $(500 \mu \mathrm{m})$, acquired on the TESCAN MIRA 3 FE-SEM equipped with a rocking beam setup; the acquisition parameters were: acceleration voltage $V=30 \mathrm{kV}$, opening angle of the incident beam cone, $\theta_{c}=14.35^{\circ}$, working distance, $6.2 \mathrm{~mm}$. A pattern fit resulted in the following Euler angles: $\left(\varphi_{1}, \Phi, \varphi_{2}\right) \equiv\left(121.1^{\circ}, 46.2^{\circ}, 203.7^{\circ}\right)$. Figure 5b shows the corresponding simulated ECP without distortion. Figures $5 \mathrm{c}$ and $5 \mathrm{~d}$ show the addition of barrel distortion and Poisson noise, respectively, to the simulated pattern. The distortion coefficient, $D=(0.4,1.0) \times 10^{-6}$ was found to give the best match between experimental and simulated patterns; it is clear from a visual comparison that barrel distortion alone does not fully reproduce the distortions present in the experimental pattern. An adaptive histogram equalization (Pizer et al., 1987) has been performed on both the experimental and simulated patterns to enhance and match the contrast. Note that there is significant bending of the Kikuchi bands close to the border of the pattern. We shall see later that even though the noise and distortion are important for improving the overall agreement between simulated and experimental patterns, the dictionary approach is relatively insensitive to these second-order corrections.

\section{Estimating Detector Parameters}

For the dictionary approach to work correctly, the right set of geometric detector parameters for a particular experiment must be estimated. For ECPs, the relevant parameters are the working distance, the sample tilt and the incident beam cone semi-angle. The problem of finding the correct set of detector parameters can be reformulated in terms of an optimization problem, where the best set of parameters will maximize either the mutual information or the dot product between the simulated and experimental images (Goshtasby, 2012). As the dependence of these metrics on the detector parameters is not known analytically, DFO algorithms are well suited to this optimization problem. The performance of a number of DFO algorithms, in terms of finding the global optimum and refining near optimal solutions, has been documented in (Rios \& Sahinidis, 2013). For the present paper, two of these algorithms, namely the Nelder-Mead simplex (Nelder \& Mead, 1965) and the bound optimization by quadratic approximation (BOBYQA) (Powell, 2009) were evaluated. It is also worthwhile to note that further refinement of the Euler angles obtained from the dictionary approach can also be performed using this method, with the Euler angles obtained from the dictionary method serving as a starting point for the refinement algorithm. The details of obtaining the detector parameters using dynamically simulated diffraction patterns will be published elsewhere.

\section{Dictionary Generation and Indexing}

The dictionary approach requires a uniform sampling of orientation space. This is essential so that different regions of $\mathrm{SO}(3)$ are represented with equal weight in the pattern dictionary. It is also important to note that for a given crystal structure, only the relevant FZ needs to be sampled as all possible unique orientations for the given crystal symmetry are represented by the FZ. For the present study, we will use the Rodrigues FZ because, unlike in other orientation representations, the $\mathrm{FZ}$ in Rodrigues space has planar boundaries and is convenient for sampling. Although there are other sampling methods in the literature, such as the Hopf method described in Yershova et al. (2010) and the HEALPix framework described in Górski et al. (2005), it is not straightforward to adapt them for integration with crystallographic symmetry. In this study, we have employed the cubochoric sampling method (Roşca et al., 2014), which is an equal-volume mapping of a uniform three-dimensional (3D) cubic grid onto a grid on the unit quaternion sphere. Thus, the sampling is uniform in the equal-volume sense. 

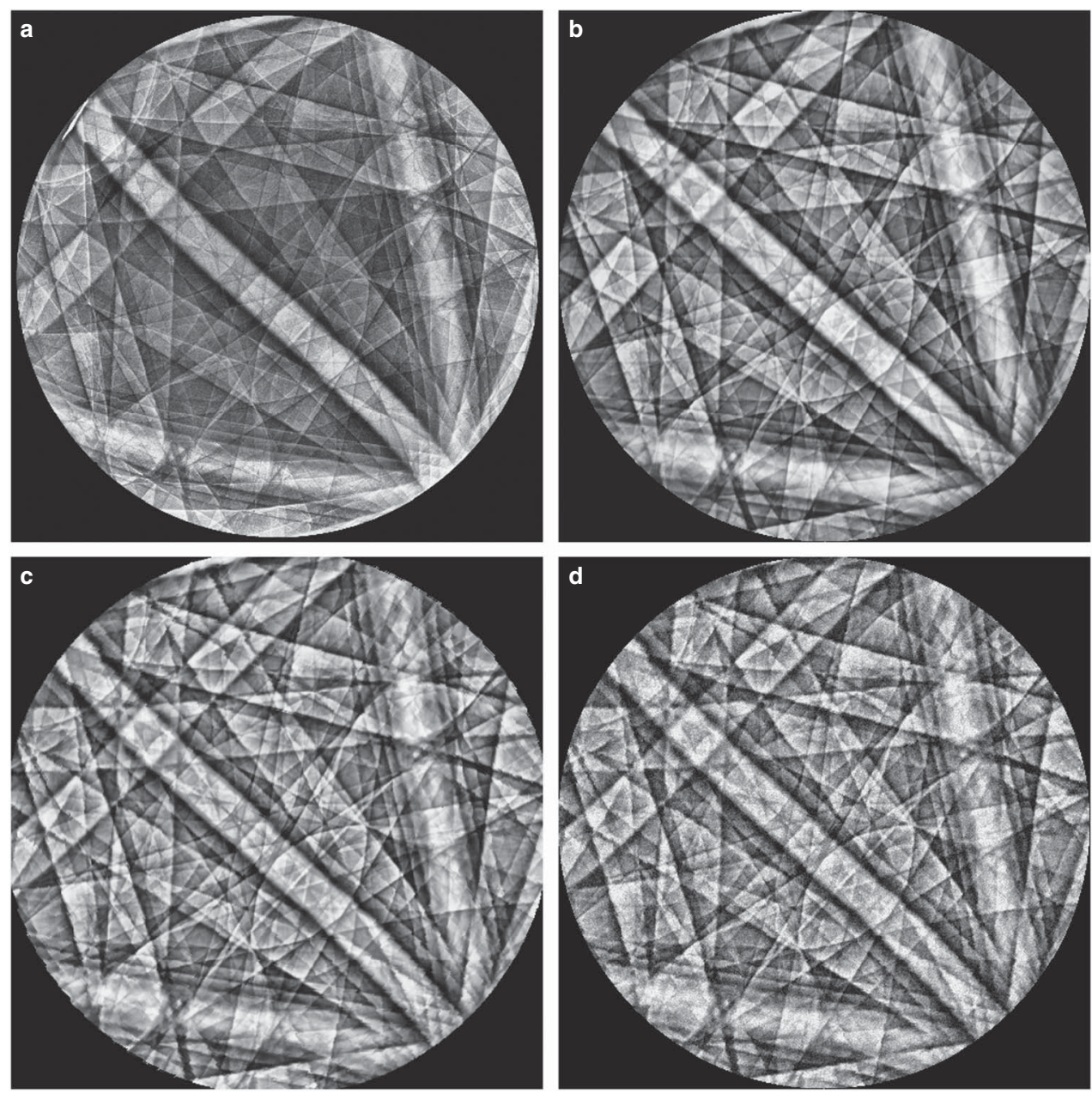

Figure 5. a: Experimental channeling pattern. b: Simulated channeling pattern for correct detector parameters and Euler angle triplet. c: Distortion added to the simulated pattern (d) both distortion and Poisson noise added to simulated pattern. The contrast for both experimental and simulated patterns have been enhanced using the adaptive histogram equalization algorithm.

This cubochoric mapping is uniform, hierarchical, and isolatitudinal, and is carried out in three steps:

- Generate a uniform grid inside a cube of edge length $\pi^{2 / 3}$. With $N$ being the number of sampling points along a semiedge of the cube, there are two choices for the grid. The first choice, $S_{000}$ is a grid of $(2 N+1)^{3}$ points containing the identity orientation. The other choice is the dual grid, $S_{111}$ which has $8 N^{3}$ points and does not contain the identity orientation. The fineness of the resulting orientation sampling depends on the value of $N$ (Roşca et al., 2014).

- Mapping the uniform grid on the cube to a uniform grid on an homochoric ball of radius $(3 \pi / 4)^{1 / 3}$. This is done by dividing the cube into six square pyramids with apex at the center of the cube and mapping each pyramid to a sextant of the ball (Roşca \& Plonka, 2011).

- Finally, the uniform grid on the ball is mapped onto the Northern hemisphere of the unit quaternion sphere, isomorphic with the space of $3 \mathrm{D}$ orientations, using the generalization of the azimuthal equal-area Lambert projection, also referred to as the homochoric projection (Roşca et al., 2014).

The dictionary can be generated by combining all the steps in the previous section and serves as a precomputed look-up table against which the experimental patterns are matched. As the dictionary is generated on a discrete grid of orientations, it is not necessary and highly unlikely that the exact orientation corresponding to the experimental pattern is present in the dictionary. For the dictionary approach to be effective, the pattern matching step needs to pick out the pattern that is closest in misorientation to the experimental pattern. Therefore, the criterion used for pattern matching must serve as a proxy for the misorientation between the dictionary and experimental patterns. Using uniform sampling of misorientation iso-surfaces, it has been shown in Singh \& De Graef (2016) that the dot product method is a good proxy for misorientations up to about $3-5^{\circ}$ for electron backscatter diffraction patterns (EBSPs). As ECPs and EBSPs are very similar, the dot product was used as the similarity 
metric in this work as well. Each experimental channeling pattern is arranged in a column vector, $\mathbf{e}_{i}\left(i \in\left\{1,2, \ldots, N_{e}\right\}\right)$ and normalized to give unit vectors $\hat{\mathbf{e}}=\mathbf{e}_{i} /\left\|\mathbf{e}_{i}\right\|$, where $\|\cdot\|$ represents the Euclidean norm of the vector. These patterns are then matched with each normalized dictionary member, $\hat{\mathbf{d}}_{j}\left(j \in\left\{1,2, \ldots, N_{d}\right\}\right)$ using the dot product metric given by

$$
\alpha_{i j}=\hat{\mathbf{e}} \cdot \hat{\mathbf{d}}_{\mathbf{j}} .
$$

For each experimental pattern $\hat{\mathbf{e}}_{i}$, the dictionary element with the highest dot product, $\sigma_{i j}$ is considered to be the best match and the orientation corresponding to that dictionary element is assigned as the orientation for the experimental pattern. To achieve an average grid spacing of about $1.4^{\circ}$, the value of $N=100$ must be used in the $S_{000}$ grid. Even for this modest angular step size, the number of entries in the dictionary is 333,227 for cubic (octahedral) rotational symmetry. The number of patterns can very easily become greater than a few million for crystals with lower symmetry, with more than eight million entries in the absence of any symmetry (for $N=100$ ); this makes the dictionary approach computationally intensive. The approach is made computationally tractable by using a combination of OpenMP parallellization for the dictionary generation with a massively parallel graphics processing unit platform using the OpenCL approach for the computation of the dot products between the dictionary and experimental patterns.

\section{Results}

\section{Indexing and Orientation Refinement}

In the absence of a commercial automated pattern acquisition and indexing system similar to those available for EBSD analysis, we must use a different validation technique. Also, the Euler angles obtained by commercial EBSD packages cannot be compared directly to the ones obtained from our dictionary approach since they use different reference frames and FZs. Therefore, to validate our indexing method, in addition to comparing the experimental and simulated patterns for the Euler angles obtained from the dictionary method, the reconstructed misorientations between pairs of patterns having known misorientations were determined. Channeling patterns from a single grain in semiconductor grade poly-Si with large grains $(500 \mu \mathrm{m})$ were recorded on a TESCAN MIRA 3 FE-SEM equipped with a rocking beam setup, with $0^{\circ},+5^{\circ}$, and $-5^{\circ}$ sample tilts and a working distance of $9.63 \mathrm{~mm}$; the patterns are shown in Figures 6a and $6 \mathrm{c}$. There is excellent agreement between the experimental and simulated patterns. The opening angle of the cone was obtained using the BOBYQA algorithm and found to be $11.42^{\circ}$. The Euler angles obtained from the
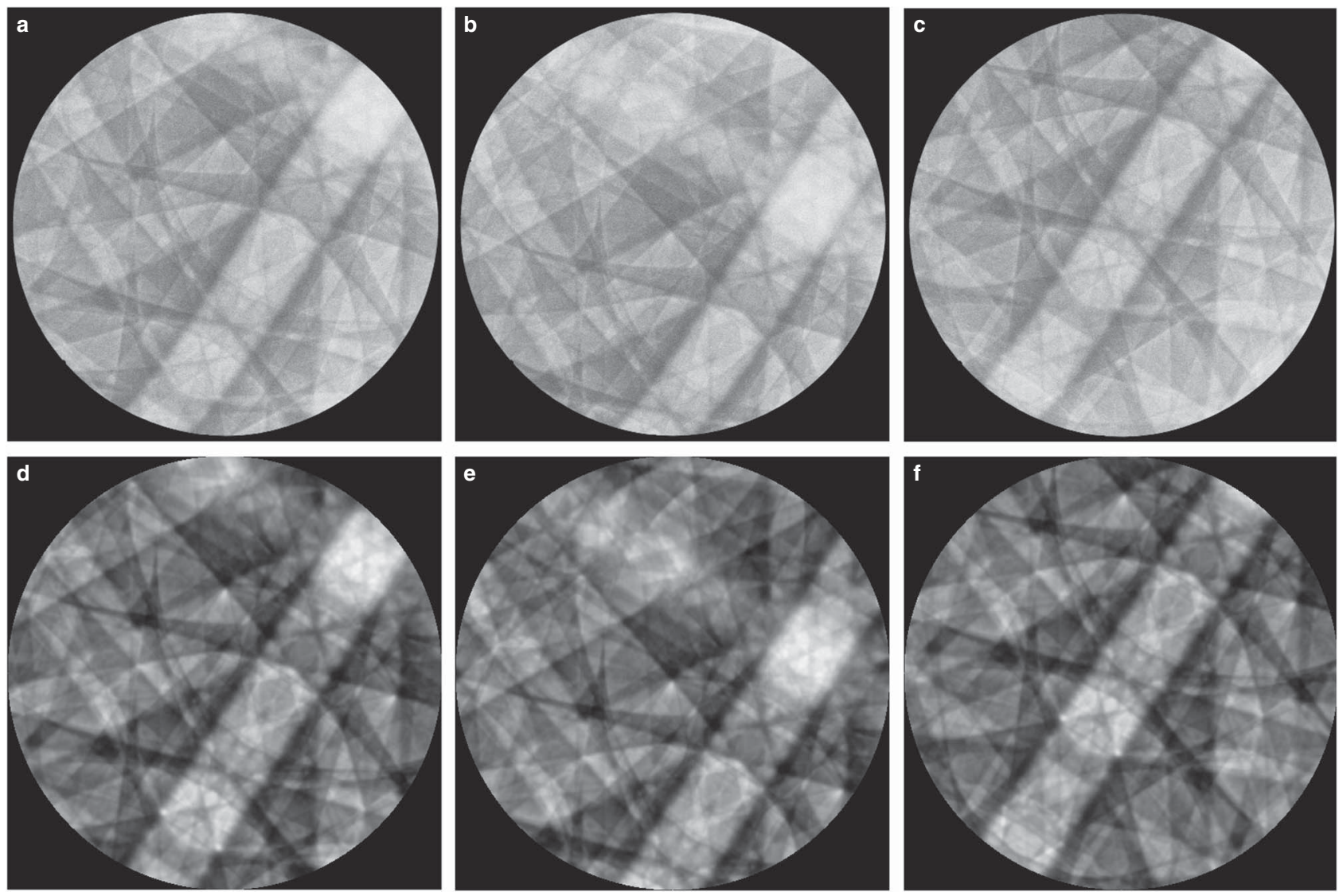

Figure 6. Experimental channeling patterns for (a) $0^{\circ}(\mathbf{b})+5^{\circ}$, and (c) $-5^{\circ}$ stage tilt. d-f: corresponding simulated channeling patterns for $(\mathbf{a}-\mathbf{c})$. Detector parameters and Euler angles in text and Table 1. 
dictionary approach and the angles after further refinement are shown in Table 1.

The orientation refinement was also performed using the BOBYQA DFO algorithm. The simulated patterns for the refined Euler angles are shown in Figures $6 \mathrm{~d}$ and $6 \mathrm{f}$. No noise or distortions were applied and the intensity of the simulated patterns was rescaled to match the experimental patterns. The misorientation between pairs of Euler angles obtained from the dictionary and after refinement is shown in Table 2. The misorientations for the Euler angles from the dictionary approach are slightly different from the true misorientations (reported in the table). This difference can be attributed to the discrete nature of the orientation sampling such that the exact orientation was not present in the dictionary. However, after refinement, the misorientation is in near perfect agreement with the true values.

\section{Error Analysis}

The dictionary approach uses a uniformly sampled grid in orientation space to generate the dictionary. As mentioned previously, it is not necessary and highly unlikely that the experimental pattern will lie on a sampled grid point. Furthermore, the dictionary method requires the detector parameters as its input. Therefore, it is important to study the error introduced in the final indexing as a function of the sampling step size and the error in the initial detector parameters. A set of 1,000 random orientations was generated and simulated channeling patterns for Silicon at $10 \mathrm{kV}$ and $\theta_{c}=15^{\circ}$ were computed. These patterns were indexed using the dictionary approach for different sampling step sizes and different error levels in the initial detector parameters. The disorientation angle between the orientation given by the dictionary approach and the true orientations was then taken as a measure of the error. The mean disorientation error of the 1,000 patterns along with the standard deviation are reported as the efficacy of the method. It is important to note that due to the limited capture angle in a typical channeling

Table 1. Euler Triplet for the Tree Sample Tilts Obtained from the Dictionary Method and After Refinement Using the Bound Optimization by Quadratic Approximation Algorithm.

\begin{tabular}{lccc}
\hline ID & Stage Tilt $\left(^{\circ}\right)$ & Dictionary $\left(^{\circ}\right)$ & Refined $\left(^{\circ}\right)$ \\
\hline 1 & 0 & $(330.69,26.11,38.18)$ & $(333.66,26.43,38.26)$ \\
2 & +5 & $(338.85,30.91,34.85)$ & $(336.85,30.97,34.08)$ \\
3 & -5 & $(327.61,21.75,43.67)$ & $(328.51,21.96,43.89)$ \\
\hline
\end{tabular}

Table 2. Disorientation for the Euler's Triplet Pairs for the Dictionary Angles and the Refined Angles Along with the True Values.

\begin{tabular}{lccc}
\hline IDs & Dictionary $\left({ }^{\circ}\right)$ & Refined $\left(^{\circ}\right)$ & True $\left(^{\circ}\right)$ \\
\hline$(1,2)$ & 7.26 & 4.98 & 5.0 \\
$(1,3)$ & 5.26 & 5.03 & 5.0 \\
$(2,3)$ & 10.47 & 9.99 & 10.0 \\
\hline
\end{tabular}

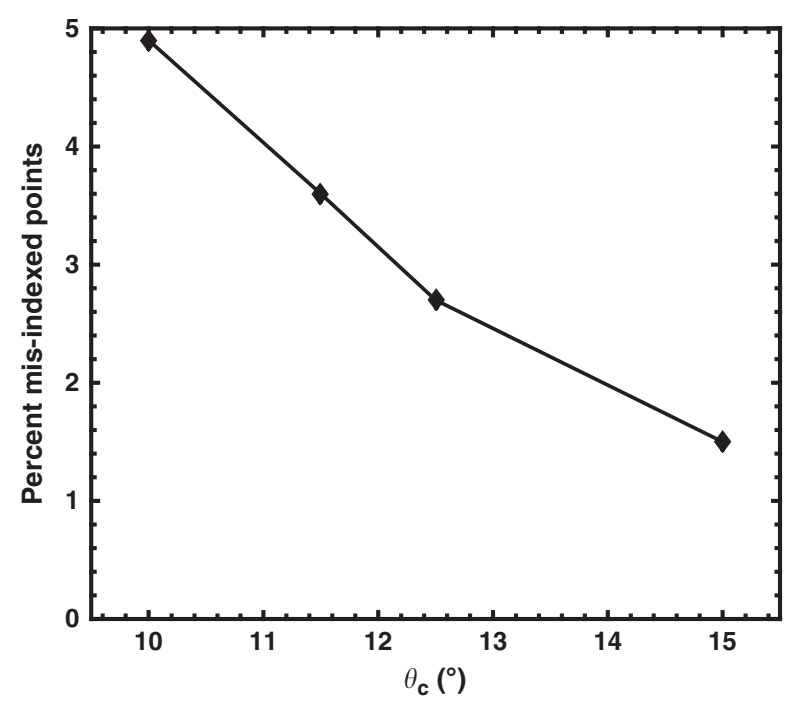

Figure 7. Percent of misindexed points as a function of the semi-capture angle.

pattern $\left(20-30^{\circ}\right)$ compared to an EBSP $\left(70-80^{\circ}\right)$, there is a small fraction of orientations which are misindexed to its pseudosymmetric orientation variant rotated by $\left[60^{\circ}(111)\right]$. This is particularly evident from the fact that the fraction of misindexed points decreases with increasing capture angle as shown in Figure 7. This problem can be completely alleviated if a sufficiently fine sampling grid is used to generate the dictionary; however, this makes the method computationally more expensive. A detailed analysis of predicting pseudosymmetric variants in a crystal with arbitrary symmetry using dynamical simulations and setting up the microscope geometry to counter such issues will be published elsewhere. For the error analysis in the present paper, we have ignored the small fraction of orientations which were misindexed.

Figure 8a shows the orientation error as a function of the number of sampling points along the cubochoric semi-edge. As the dictionary is generated at the sampling grid points, decreasing the sampling step size leads to more patterns in the dictionary. This decreases the orientation error, but increases the computational cost of the method. As shown previously, the better approach is to use a moderate sampling step size $(N \sim 100)$ and then refine the orientations using the DFO optimization algorithm. Figure $8 \mathrm{~b}$ shows the orientation error as a function of the error in the input detector parameters for $N=100$ sampling points along the cubochoric cube semiedge. For the true detector parameters, the orientation error is about $1.0^{\circ}$ with $0.5^{\circ} \mathrm{SD}$. The method is relatively insensitive to increasing error in the input detector parameters up to about $5 \%$ error. The error increases beyond that point and the method fails catastrophically for about $8 \%$ error in the input detector parameter.

\section{SUMMARY}

In this paper, we have introduced a new dictionary-based approach to indexing ECPs. The basic ingredients of this method include a GFP, which is a physics-based forward model 

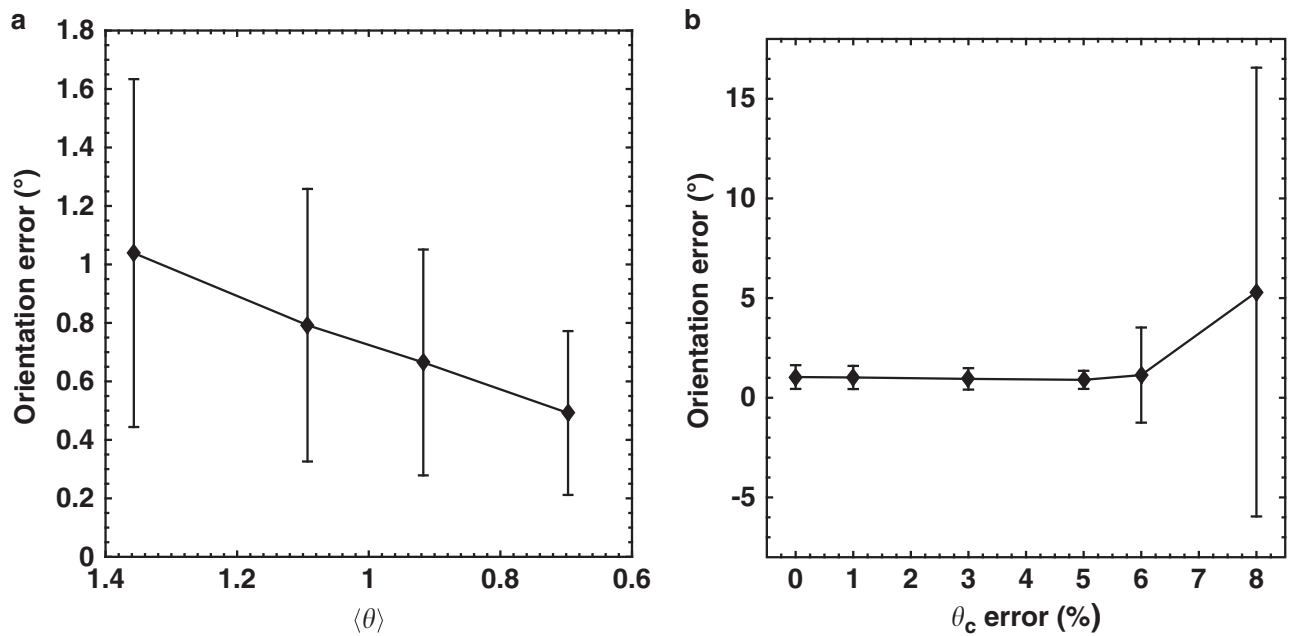

Figure 8. a: Orientation error as a function of mean sampling step size and (b) error in input detector parameters.

for the scattering process, a detector and noise model and a method to uniformly sample orientation space. The deterministic forward model is merged with stochastic MC simulations to calculate the scattering master pattern. Individual channeling patterns are extracted from the master pattern using bilinear interpolation. The cubochoric representation is introduced as a means to uniformly sample orientation space. The forward model coupled with a DFO algorithm are used to evaluate the correct detector parameters and to refine the orientations obtained from the dictionary approach. In contrast to the Hough transform-based method which extracts features from diffraction patterns, our approach uses all available pixels. Pattern matching is performed using the normalized dot product as the similarity metric. The method is applied to poly-Si as the model system. The results are in excellent agreement with the calibrated values. Finally, an error analysis is performed using a set of 1,000 simulated patterns with random orientations. The results show that the method produces a mean orientation error of $1^{\circ}$ with $0.5^{\circ} \mathrm{SD}$ for the true detector parameters and $N=100$ points along the cubchoric cube semi-edge, corresponding to a mean sampling step size of $1.4^{\circ}$. Increasing the number of sampling points decreases the error. The mean orientation error is insensitive to an error in the detector parameters up to about $5 \%$ error in detector parameter.

\section{ACKNOWLEDGMENTS}

The authors wish to acknowledge an Air Force Office of Scientific Research (AFOSR) MURI program (contract \# FA9550-12-1-0458) as well as the computational facilities of the Materials Characterization Facility at $\mathrm{CMU}$ under grant \# MCF-677785.

\section{REFERENCES}

Ahmed, J., Wilkinson, A. \& RoberTs, S. (1997). Characterizing dislocation structures in bulk fatigued copper single crystals using electron channelling contrast imaging (ECCI). Philos Mag Lett 76, 237-245.
Callahan, P. \& De Graef, M. (2013). Dynamical EBSD patterns Part I: Pattern simulations. Microsc Microanal 19, 1255-1265.

Chen, Y., Park, S.U., Wei, D., Newstadt, G., Jackson, M., Simmons, J., De Graef, M. \& Hero, A. (2015). A dictionary approach to EBSD indexing. Microsc Microanal 21, 739-752.

CoATes, D. (1967). Kikuchi-like reflection patterns obtained with scanning electron microscope. Philos Mag 16, 1179-1185.

Crimp, M., Simkin, B. \& NG, B.C. (2001). Demonstration of the $\mathrm{g} \cdot \mathrm{b} \times \mathrm{u}=0$ edge dislocation invisibility criterion for electron channelling contrast imaging. Philos Mag Lett 81, 833-837.

Czernuszka, J., Long, N., Boyes, E. \& Hirsch, P. (1990). Imaging of dislocations using backscattered electrons in a scanning electron microscope. Philos Mag Lett 62, 227-232.

De Graef, M. (2003). Introduction to Conventional Transmission Electron Microscopy. Cambridge, UK: Cambridge University Press.

Deitz, J., Carnevale, S., De Graef, M., McComb, D. \& Grassman, T. (2016). Characterization of encapsulated quantum dots via electron channeling contrast imaging. Appl Phys Lett 109, 062101.

Dudarev, S., Rez, P. \& Whelan, M. (1995). Theory of electron backscattering from crystals. Phys Rev B 51, 3397-3405.

Górski, K., Hivon, E., Banday, A., Wandelt, B., Hansen, F., Reinecke, M. \& Bartelmann, M. (2005). HeAlPix: A framework for high-resolution discretization and fast analysis of data distributed on the sphere. Astrophys J 622, 759-771.

Goshtasby, A. (2012). Image Registration, Advances in Computer Vision and Pattern Recognition. London: Springer Verlag.

Gutierrez-Urrutia, I., Zaefferer, S. \& RaAbe, D. (2013). Coupling of electron channeling with EBSD: Toward the quantitative characterization of deformation structures in the SEM. J Mater 65, 1229-1236.

Hawkes, P.W. \& Kasper, E. (1989a). Principles of Electron Optics: Basic Geometrical Optics, vol. 2, New York: Academic Press.

Hawkes, P.W. \& Kasper, E. (1989b). Principles of Electron Optics: Wave Optics, vol. 3, New York: Academic Press.

Joy, D. \& Booker, G. (1971). Simultaneous display of micrograph and selected-area channelling pattern using the scanning electron microscope. J Phys E Sci Instrum 4, 837-842.

Joy, D., Newbury, D. \& Davidson, D. (1982). Electron channeling patterns in the scanning electron microscope. J Appl Phys 53, 81-122.

Mansour, H., Crimp, M., Gey, N. \& Maloufi, N. (2015). Accurate electron channeling contrast analysis of a low angle sub-grain boundary. Scr Mater 109, 76-79. 
Marthinsen, K. \& Høier, R. (1986). Many-beam effects and phase information in electron channeling patterns. Acta Cryst A 42, 484-492.

Nelder, J. \& Mead, R. (1965). A simplex method for function minimization. Comput J 7, 308-313.

Picard, Y., Liu, M., Lammatao, J., Kamaladasa, R. \& De Graef, M. (2014). Theory of dynamical electron channeling contrast images of near-surface crystal defects. Ultramicroscopy 146, 71-78.

Picard, Y. \& Twigg, M. (2008). Diffraction contrast and Bragg reflection determination in forescattered electron channeling contrast images of threading screw dislocations in $4 \mathrm{~h}-\mathrm{SiC}$. J Appl Phys 104, 124906.

Pizer, S., Amburn, E., Austin, J., Cromartie, R., Geselowitz, A., Greer, T., Romney, B., Zimmerman, J. \& Zuiderveld, K. (1987). Adaptive histogram equalization and its variation. Comput Vision, Graph Image Process 39, 355-368.

Powell, M. (2009). The BOBYQA algorithm for bound constrained optimization without derivatives, Technical Report. Department of Applied Mathematics and Theoretical Physics, Cambridge University.

Rios, L. \& SAHINIDIS, N. (2013). Derivative-free optimization: A review of algorithms and comparison of software implementations. J Global Optim 56, 1247-1293.

RoşcA, D. (2010). New uniform grids on the sphere. Astron Astrophys 520, A63.

Roşca, D. \& Plonka, G. (2011). New uniform grids on the sphere. J Comput Appl Math 236, A63.

Roşca, D., Morawiec, A. \& De Graef, M. (2014). A new method of constructing a grid in the space of 3D rotations and its applications to texture analysis. Model Simul Mater Sci Eng 22, 075013.

Rossouw, C., Miller, P., Josefsson, T. \& Allen, L. (1994). Zone axis backscattered electron contrast for fast electrons. Philos Mag A 70, 985-998.
Schmidt, N. \& Olesen, N. (1989). Computer aided determination of crystal lattice orientation from electron channeling patterns in the SEM. Can Mineral 27, 15-22.

Singh, S. \& De Graef, M. (2016). Orientation sampling for dictionary-based diffraction pattern indexing methods. Model Simul Mater Sci Eng 159, 81-94.

Spencer, J. \& Humphreys, C. (1980). A multiple-scattering transporttheory for electron channeling patterns. Philos Mag A 42, 433-451.

SzILAgyi, M. (1988). Electron and Ion Optics. New York: Plenum Press.

Trager-Cowan, C., Sweeney, F., Trimby, P., Day, A., Gholinia, A., Schmidt, N., Parbrook, P., Wilkinson, A. \& Watson, I.M. (2007). Electron backscatter diffraction and electron channeling contrast imaging of tilt and dislocations in nitride thin films. Phys Rev B 75, 085301.

VAN Essen, C. \& SChulson, E. (1969). Selected area channelling patterns in the scanning electron microscope. J Mater Sci 4, 336-339.

Van Essen, C., Schulson, E. \& Donaghay, R. (1970). Electron channelling patterns from small $\left(10^{\wedge} \mathrm{m}\right)$ selected areas in the scanning electron microscope. Nature 255, 847-848.

Winkelmann, A., Schröter, B. \& Richter, W. (2003). Dynamical simulations of zone axis electron channelling patterns of cubic silicon carbide. Ultramicroscopy 98, 1-7.

Wright, S., Nowell, M., Lindeman, S., Camus, P., De Graef, M. \& JACKSON, M. (2015). Introduction and comparison of new EBSD post-processing methodologies. Ultramicroscopy 159, 81-94.

Yershova, A., Jain, S., LaValle, S. \& Mitchell, J. (2010). Generating uniform incremental grids on $\mathrm{SO}(3)$ using the Hopf fibration. Int J Robot Res 29, 801-812.

ZaefFerer, S. \& Elhami, N. (2014). Theory and application of electron channelling contrast imaging under controlled diffraction conditions. Acta Mater 75, 20-50. 